\title{
Anti-malarial effect of novel chloroquine derivatives as agents for the treatment of malaria
}

Seon-Ju Yeo ${ }^{1 \dagger}$, Dong-Xu Liu ${ }^{1 \dagger}$, Hak Sung Kim² ${ }^{*}$ and Hyun Park ${ }^{1^{*}}$

\begin{abstract}
Background: The widespread emergence of anti-malarial drug resistance has necessitated the discovery of novel anti-malarial drug candidates. In this study, chloroquine derivatives were evaluated for the improved anti-malarial activity.

Results: Novel two derivatives (SKM13 and SKM14) were synthesized based on the chloroquine (CQ) template containing modified side chains such as a, $\beta$-unsaturated amides and phenylmethyl group. The selective index indicated that SKM13 was 1.28-fold more effective than CQ against the CQ-resistant strain Plasmodium falciparum. An in vivo mouse study demonstrated that SKM13 $(20 \mathrm{mg} / \mathrm{kg})$ could completely inhibit Plasmodium berghei growth in blood and increased the survival rate from 40 to 100\% at 12 days after infection. Haematological parameters [red blood cell (RBC) count, haemoglobin level, and haematocrit level] were observed as an indication of clinical malarial anaemia during an evaluation of the efficacy of SKM13 in a 4-day suppression test. An in vivo study showed a decrease of greater than 70\% in the number of RBC in P. berghei-infected mice over 12 days, but the SKM13 $(20 \mathrm{mg} / \mathrm{kg})$-treated group showed no loss of RBC.
\end{abstract}

Conclusions: $C Q$ derivatives with substituents such as $\alpha, \beta$-unsaturated amides and phenylmethyl group have enhanced anti-malarial activity against the CQ-resistant strain P. falciparum, and SKM13 is an excellent anti-malarial drug candidate in mice model.

Keywords: Chloroquine derivatives, SKM13, Anti-malarial efficacy, a, $\beta$-unsaturated amides

\section{Background}

Malaria is a disease affecting humans, caused by a protozoan parasite of the Plasmodium genus, with 107 countries and territories having areas at risk of transmission [1]. The World Health Organization (WHO) reported the occurrence of 214 million cases worldwide in 2015 , and the death of 438,000 people, mostly children in the African region [2].

\footnotetext{
*Correspondence: hankidad@wku.ac.kr; hyunpk@wku.ac.kr

†Seon-Ju Yeo and Dong-Xu Liu contributed equally to this work

${ }^{1}$ Department of Infection Biology, School of Medicine, Zoonosis Research

Center, Wonkwang University, 460, Iksan-daero, Iksan, Jeolabuk-do 54538,

Republic of Korea

${ }^{2}$ College of Pharmacy, Institute of Pharmaceutical Research

and Development, Wonkwang University, 460, Iksan-daero, Iksan,

Jeolabuk-do 54538, Republic of Korea
}

Successful malaria control in the past decade was dependent on treatment with efficacious anti-malarial drugs [3]. Quinoline drugs such as chloroquine (CQ) and quinine were the cornerstone of malaria treatment [4]. Quinine was used as anti-malarial medication from the seventeenth century until the 1920 s, when CQ, a more effective synthetic anti-malarial, became available [5]. However, the extensive use of CQ led to the development of a chloroquine-resistant malaria parasite Plasmodium falciparum in Southeast Asia, Oceania, and South America in the late 1950s and early 1960s [6]. This drug resistance inspired a significant effort throughout the twentieth century to identify new anti-malarial agents for the improvement of global public health. There is still a need to produce "novel" drugs with different properties, which has led to dramatic changes in the way new targets are identified [7]. 
Although the molecular basis of chloroquine action is yet to be properly elucidated, the mechanism has traditionally been considered to occur through interference in the haemozoin crystal formation of the Plasmodium species, leading to detoxification of the malaria parasite $[8,9]$. Chloroquine-resistant $P$. falciparum survives by reduction of drug accumulation in the digestive vacuole. In addition to being effective as an anti-malarial medication, CQ has emerged as a prospective adjunct with antiviral effects [10, 11], antitumour activity [12], and as an effector of cell-death by altering lysosomal function [13].

More efficacious drugs are currently available [5]. For example, artemisinin has been reported as a potent anti-malarial drug, but the emergence of resistance has increased the failure rate of artemisinin-based combination therapy [14-16]. As resistance to existing drugs develops, new drugs need to be introduced; for P. falciparum, the use of a combination of several drugs with different modes of action is recommended to provide an adequate cure rate and delay the development of resistance.

Several novel drug candidates based on the CQ structure, with modifications of both the side chain and the quinoline ring, have been reported [17-19]. In a previous study, the Michael-acceptor role of the $\alpha, \beta$-unsaturated amide, which mimics the functional group present in gallinamide A and many anti-malarial chalcones, was found to stabilize the thiolate covalent bond between calpain, a cysteine protease required for cell cycle progression in Plasmodium parasites [20], and the $\beta$ carbon of the $\alpha, \beta$ unsaturated amide [21, 22].

In the present study, two novel derivatives were designed based on the CQ structural template with a modified side chain, such as $\alpha, \beta$-unsaturated amides and phenylmethyl group. These two derivatives were evaluated for anti-malarial activity in vitro and in vivo.

\section{Methods}

\section{Reagents}

Chloroquine and atovaquone were purchased from Sigma Aldrich (St. Louis, USA). SYTOX ${ }^{\circledR}$ Green nucleic acid stain was purchased from Life Technologies (Carlsbad, USA). The CellTiter $96^{\circledR}$ AQueous One Solution reagent was purchased from Promega (Madison, USA).

\section{Synthesis of SKM13 and SKM14}

SKM13 and SKM14 were synthesized using the following scheme: (1) the coupling of 4,7-dichloroquinoline and phenylalanine [23]; (2) the formation of Weinreb amide [24]; (3) reduction to aldehyde [25]; (4) Horner-Wadsworth-Emmons reaction with the amide phosphonate [26]. The chemical structures were confirmed by proton NMR.

\section{In vitro culture of Plasmodium species}

Plasmodium falciparum 3D7 (American Type Culture Collection, ATCC PRA-405D) and P. falciparum FCR3 (American Type Culture Collection, ATCC ${ }^{\circledR}$ 30932) were purchased from the ATCC (Manassas, USA). The chloroquine-susceptible strain Plasmodium berghei NK65 (MRA-268) and the atovaquone-resistant strain P. berghei NAT (MRA-415) were purchased from Bei Resources (Manassas, USA). The P. falciparum strains 3D7 and FCR3 were grown in human erythrocytes as previously described [27]. Briefly, parasites were maintained in continuous culture with $5 \%$ haematocrit of type $\mathrm{O}$ human red blood cells suspended in Roswell Park Memorial Institute (RPMI) 1640 medium supplemented with $24 \mathrm{mM} \mathrm{NaHCO}, 25 \mathrm{mM}$ HEPES, 0.8\% hypoxanthine, $0.9 \%$ Albumax, and $25 \mu \mathrm{g} / \mathrm{mL}$ of gentamicin. The 6-well plates were placed in an incubator (atmosphere: $5 \% \mathrm{CO}_{2}, 5 \% \mathrm{O}_{2}$, and $90 \% \mathrm{~N}_{2}$ ) at $37{ }^{\circ} \mathrm{C}$ and the medium was changed daily when the level of parasitaemia was at least $5 \%$. The parasite density was determined by Giemsa staining of thin smears and expressed as a percentage of infected erythrocytes in a field of a total of 500 erythrocytes.

\section{In vitro anti-malarial activity by FACS analysis}

To assess the effect of the compounds on malarial parasite growth, the parasites were seeded in 48-well plates at a density of 0.5 in $2 \%$ haematocrit. The CQ compounds were then serially diluted in medium and incubated with the parasites for $48 \mathrm{~h}$ without any medium change. Finally, $100 \mu \mathrm{L}$ of the $P$. falciparum cultures were withdrawn from each well of the 48 -well plate and $1 \mu \mathrm{L}$ of the blood pellet was mixed in $5 \mathrm{mM}$ SYTOX green solution to obtain a final volume of $1.5 \mathrm{~mL}$. The mixture was left to stand in the dark for $30 \mathrm{~min}$ at room temperature and the anti-malarial activity of the compounds was analysed by fluorescence-activated cell sorting (FACS) using a FACS Calibur flow cytometer (BD Biosciences, Franklin Lakes, USA).

\section{In vitro cytotoxicity}

The MDCK cells were purchased from the Korean Cell Line Bank. The cells were seeded in a 96-well plate in Dulbecco's modified Eagle's medium (DMEM) supplemented with $10 \%$ fetal bovine serum (FBS) and incubated with serially diluted CQ compounds for $48 \mathrm{~h}$.

After the incubation period, $20 \mu \mathrm{L}$ of CellTiter $96^{\circledR}$ AQueous One Solution reagent was added to each well. After incubation for $1 \mathrm{~h}$ at $37^{\circ} \mathrm{C}$ in a humidified $5 \% \mathrm{CO}_{2}$ atmosphere, the absorbance was measured at $490 \mathrm{~nm}$ using an enzyme-linked immunosorbent assay (ELISA) plate reader. 


\section{Test for suppressive activity (Peter's 4-day test)}

ICR female mice (Orient Bio Co., Seongnam, South Korea), 7 weeks old, were housed at Wonkwang University. All mice were bred and maintained under constant conditions. Animal experiments were performed using an experimental protocol approved by the Animal Care and Use Committee at Wonkwang University (WKU16-40).

The atovaquone-resistant strain $P$. berghei NAT and the chloroquine-sensitive strain $P$. berghei NK65 were used to set up an infected mouse model. The parasites kept in liquid nitrogen were thawed at $37{ }^{\circ} \mathrm{C}$ and maintained by the serial passage of blood from mouse to mouse. In this study, each mouse was injected with $10^{7}$ P. bergheiinfected erythrocytes $/ 100 \mu \mathrm{L}$ by intraperitoneal (i.p.) injection. At one day post-infection (dpi), drug treatment with SKM13 $(20 \mathrm{mg} / \mathrm{kg})$ was commenced. Atovaquone $(4 \mathrm{mg} / \mathrm{kg})$ and chloroquine $(10 \mathrm{mg} / \mathrm{kg})$ were intravenously (i.v.) administered once a day for four consecutive days as the control drug. Each day, tail blood was collected for FACS assessment of parasitaemia. Mouse weight and survival was checked every day, and haematology assays were conducted at days 3, 6, 9, and $12 \mathrm{dpi}$.

\section{Haematological assay}

The haematological testing was conducted using a fully automated blood cell counter LC-660 (HORIBA Medical, Seoul, South Korea). The parameters evaluated included white blood cell count, red blood cell count, haemoglobin, and haematocrit.

\section{Statistical analysis}

Results were statistically analysed using GraphPad Prism software 5.0. The experiments were compared using oneway ANOVA followed by Bonferroni's multiple comparison test. All results are expressed as mean \pm SD (standard deviation of the mean). $P$ values of less than 0.05 were considered statistically significant.

\section{Results}

Characterization of SKM13 and SKM14

Two novel CQ derivatives (SKM13 and SKM14) were synthesized in this study (Fig. 1). CQ and two CQ derivatives have two differences of chemical structure. In the structure of $\mathrm{CQ}$, there is a small methyl group on the side chain [28] but two CQ derivatives have phenylmethyl group derived from phenylalanine at the same position. Besides, both CQ derivatives have $\alpha, \beta$-unsaturated amide instead of methyl group of $C Q$. A little difference of chemical structure between SKM13 and SKM14 indicates that the $\alpha, \beta$-unsaturated amide in SKM13 is shorter than that in SKM14.

\section{In vitro anti-malarial activity}

After 48-h exposure to drugs, the standard in vitro assays for anti-parasitic effects were determined by FACS analysis. It showed that SKM13 and SKM14 had higher $\mathrm{IC}_{50}$ values than $\mathrm{CQ}$; $\mathrm{IC}_{50}$ values of $0.014 \pm 0.002$ (mean $\pm \mathrm{SD}$ ) $\mu \mathrm{M}, 0.17 \pm 0.01$ and $0.23 \pm 0.01 \mu \mathrm{M}$ for CQ, SKM13, and SKM14, respectively, against CQ-susceptible $P$. falciparum (3D7) (Table 1), In MDCK cells, the $\mathrm{CC}_{50}$ value indicated that SKM13 and SKM14 were more cytotoxic than CQ. Finally, it turned out that SKM13 and SKM14 had much lower selective index $(\mathrm{SI})$ value $\left(\mathrm{CC}_{50} / \mathrm{IC}_{50}\right)$ than that CQ. In contrast, the $\mathrm{IC}_{50}$ values for CQ-resistant P. falciparum (FCR3) confirmed the higher efficacy of SKM13 than CQ: CQ, SKM13, and SKM14 had $\mathrm{IC}_{50}$ values of $0.62 \pm 0.04$ (mean $\pm \mathrm{SD}$ ) $\mu \mathrm{M}, 0.37 \pm 0.01$ and $0.59 \pm 0.02 \mu \mathrm{M}$, respectively. Therefore, the $\mathrm{IC}_{50}$ values of SKM13 and SKM14 were 0.6- and 0.84-fold lower than that of CQ against CQ-resistant P. falciparum (FCR3), indicating that CQ derivatives were more effective than CQ as anti-malarial. Finally, the SI values showed that SKM13 was 1.28 -fold more effective than CQ against CQ-resistant P. falciparum. In contrast, SI value of SKM14 was lower than that of CQ.

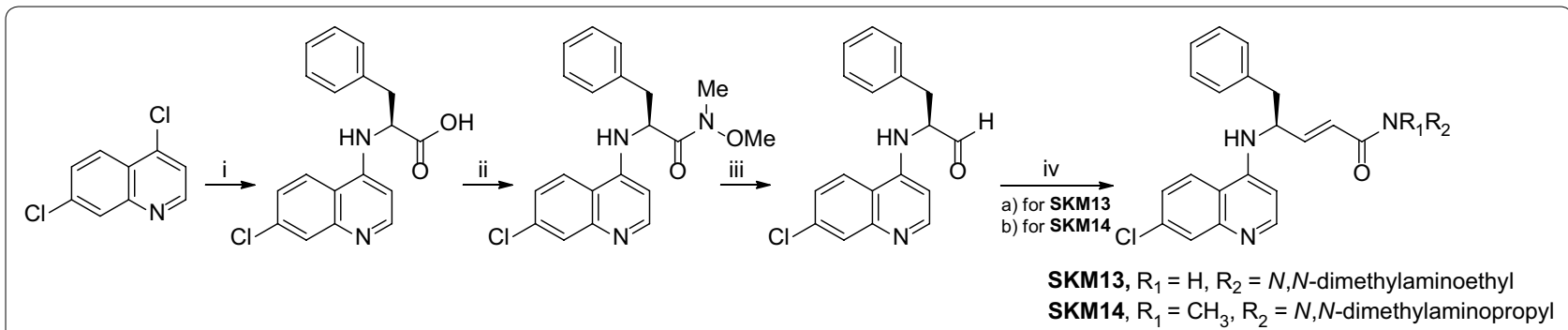

Fig. 1 Synthesis of SKM13 and SKM14. Reaction conditions: $i$ L-phenylalanine, phenol, $140{ }^{\circ} \mathrm{C}, 1 \mathrm{~h}, 55 \%$. ii N,O-dimethylhydroxylamine. $\mathrm{HCl}$, EDAC.HCl, DMAP, TEA, DCM, rt, overnight, $72 \%$. iii DIBAL, $-40^{\circ} \mathrm{C}, \mathrm{DCM}, 5$ h, $95 \%$ iv phosphonate, DCM, $\mathrm{K}^{\mathrm{t}} \mathrm{OBu}, 0^{\circ} \mathrm{C}$; (a) for SKM13 $(71 \%)$, diethyl (2-((2-(dimethylamino)ethyl)(methyl)amino)-2-oxoethyl)phosphonate; (b) for SKM14 (70\%), diethyl (2-((3-(dimethylamino)propyl)amino)-2-oxoethyl)phosphonate 
Table 1 In vitro anti-malarial activity

\begin{tabular}{lllcccc}
\hline Strain & Compound & $\left(\mathbf{I C}_{\mathbf{5 0}}, \boldsymbol{\mu M}\right)$ & Relative $\mathbf{I C}_{\mathbf{5 0}}$ & $\left.\mathbf{( C C}_{\mathbf{5 0}}, \boldsymbol{\mu M}\right)$ & Selective index $(\mathbf{S I})\left(\mathbf{C C}_{\mathbf{5 0}} / \mathbf{I C}_{\mathbf{5 0}}\right)$ & $\mathbf{R e l a t i v e} \mathbf{S I}$ \\
\hline $3 \mathrm{D} 7$ & Chloroquine & $0.014 \pm 0.002$ & 1.00 & $121.90 \pm 8.51$ & 8707.10 & 1.00 \\
& SKM13 & $0.17 \pm 0.01$ & 12.14 & $93.11 \pm 5.56$ & 547.70 & 0.06 \\
& SKM14 & $0.23 \pm 0.01$ & 16.42 & $47.06 \pm 3.21$ & 204.16 & 0.02 \\
\multirow{2}{*}{ FCR3 } & Chloroquine & $0.62 \pm 0.04$ & 1.00 & $121.90 \pm 8.51$ & 196.60 & 1.00 \\
& SKM13 & $0.37 \pm 0.01$ & 0.60 & $93.11 \pm 5.36$ & 251.70 & 1.28 \\
& SKM14 & $0.52 \pm 0.02$ & 0.84 & $47.06 \pm 3.21$ & 90.50 & 0.46 \\
\hline
\end{tabular}

Effects of chloroquine derivatives on the proliferation of CQ-susceptible and resistant strains of $P$. falciparum

Chloroquine-susceptible (3D7) and -resistant (FCR3) P. falciparum strains were cultured in the presence of increasing concentrations of CQ or each of the CQ derivatives. The values shown are the mean \pm standard deviation (SD) from three independent experiments performed in triplicate

FACS analysis results can be found in the Additional file 1: Figures S1-S6).

\section{Evaluation of the suppressive activity of SKM13 (Peter's 4-day test)}

The anti-malarial activity of SKM13 in a rodent model was assessed by a 4-day suppression test of $20 \mathrm{mg} / \mathrm{kg}$ of SKM13 in mice. As seen in Fig. 2, CQ-susceptible $P$. berghei NK65 parasites $\left(10^{7} /\right.$ mouse) were i.p. injected into ICR mice. After one day, SKM13 $(20 \mathrm{mg} / \mathrm{kg}$, in doses of $10 \mathrm{mg} / \mathrm{kg}$, twice daily) and CQ (10 mg/kg, once daily) were i.v. administered for four consecutive days.

In the parasite-infected mice, a rise in parasitaemia was observed at $2 \mathrm{dpi}$, which decreased until $7 \mathrm{dpi}$. After 8 dpi, parasitaemia increased exponentially and high parasitaemia (about 30\%) was observed until 12 dpi (Fig. 2b). Parasitaemia in the SKM13-treated group (Fig. 2c) was significantly suppressed compared to that reported for the parasite-infected group $(P<0.05)$, and the CQtreated group (Fig. 2d) showed no parasitaemia.

Body weight decreased in the parasite-infected mice. Normal body weight $(27.09 \pm 1.01 \mathrm{~g}$, mean $\pm \mathrm{SD})$ significantly decreased $(25.58 \pm 0.93 \mathrm{~g})$ at $2 \mathrm{dpi}(P<0.01)$, but remained unchanged in the SKM13-treated group $(26.96 \pm 0.56 \mathrm{~g})$ at $12 \mathrm{dpi}$, demonstrating that SKM13 $(20 \mathrm{mg} / \mathrm{kg})$ was effective in the maintenance of normal mouse metabolism until $12 \mathrm{dpi}$. CQ $(10 \mathrm{mg} / \mathrm{kg})$ also maintained normal mouse body weight.

Analysis of survival rate showed that SKM13 was effective in treating $P$. berghei infection. The parasite-infected group showed $20 \%$ mortality at $7 \mathrm{dpi}, 40 \%$ mortality at $9 \mathrm{dpi}$, and $60 \%$ mortality at $12 \mathrm{dpi}$. SKM13 treatment resulted in $100 \%$ survival rate in mice at $12 \mathrm{dpi}$ (Fig. 2e).

To assess the anti-malarial activity of SKM13 against drug-resistant $P$. berghei, atovaquone-resistant $P$. berghe $i$ NAT parasites $\left(10^{7} /\right.$ mouse) were i.p. injected into ICR mice. After 1 day, SKM13 $(20 \mathrm{mg} / \mathrm{kg}$, in doses of $10 \mathrm{mg} /$ $\mathrm{kg}$, twice daily), atovaquone (4 mg/ $\mathrm{kg}$, once daily), and CQ $(10 \mathrm{mg} / \mathrm{kg}$, once daily), were separately i.v. administered for 4 consecutive days.
At 3 dpi, parasitaemia increased in the parasite-infected group and reached a peak at $8 \mathrm{dpi}$. After $8 \mathrm{dpi}$, a plateau in the level of parasitaemia was observed until $12 \mathrm{dpi}$ (Fig. 3b). Parasitaemia in the SKM13-treated group (Fig. 3c) and CQ-treated group (Fig. 3d) was significantly suppressed compared to that reported for the parasite group $(P<0.05)$, which showed no parasitaemia. As anticipated, atovaquone $(4 \mathrm{mg} / \mathrm{kg}$ ) was unable to suppress the parasitaemia of atovaquone-resistant $P$. berghei NAT (Fig. 3e).

The normal body weight significantly decreased $(P<0.01)$ at $6 \mathrm{dpi}$ but remained unchanged until $12 \mathrm{dpi}$ in the SKM13-treated group. CQ $(10 \mathrm{mg} / \mathrm{kg})$ maintained the body weight of the infected mice. In the atovaquoneresistant $P$. berghei NAT-infected group, the mortality rate at $7 \mathrm{dpi}$ was 20 and $60 \%$ at $12 \mathrm{dpi}$. In contrast, the SKM13-treated group showed 100\% survival rate until 12 dpi (Fig. 3f).

\section{Effect of SKM13 on blood cell parameters}

Changes in blood cell count are characteristic of Plasmodium infection and haematological changes during the course of a malaria infection, such as anaemia, thrombocytopaenia, leukocytosis, or leucopenia, are well recognized [29]. In this study, WBC counts (mean \pm SD) in normal ICR mice were $8.86 \pm 1.86\left(10^{3} / \mu \mathrm{L}\right)$, which were in agreement with that reported previously [30]. Both $P$. berghei NK65 and NAT strains significantly increased WBC counts $(P<0.05)$ at 6 dpi and progressively increased WBC counts were observed until $12 \mathrm{dpi}$ $(P<0.001)$. In contrast, SKM13 or CQ treatment did not affect WBC counts (Fig. 4a). In addition to WBC counts, several other factors were observed as major indicators of clinical malaria anaemia in the efficacy evaluation of SKM13. Both P. berghei NK65 and NAT strains showed a large decrease in these values, with greater than $70 \%$ reduction in the main haematological parameters ( $\mathrm{RBC}$, haemoglobin, and haematocrit) for 12 days. However, SKM13 treatment in the 4-day suppression test did not alter the three parameters involved in anaemia $(P<0.001)$ (Fig. 4b-d). 


\section{a}

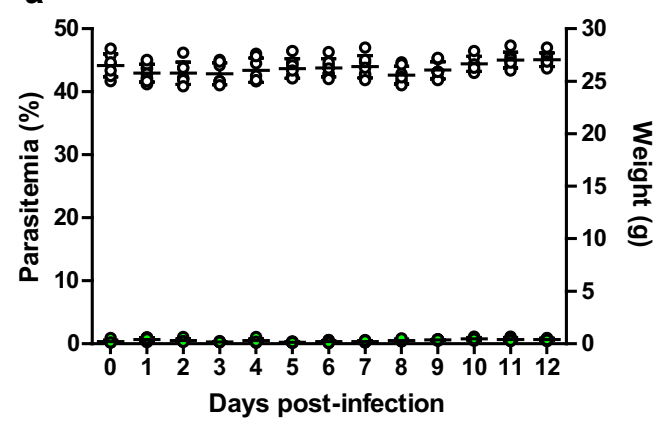

C

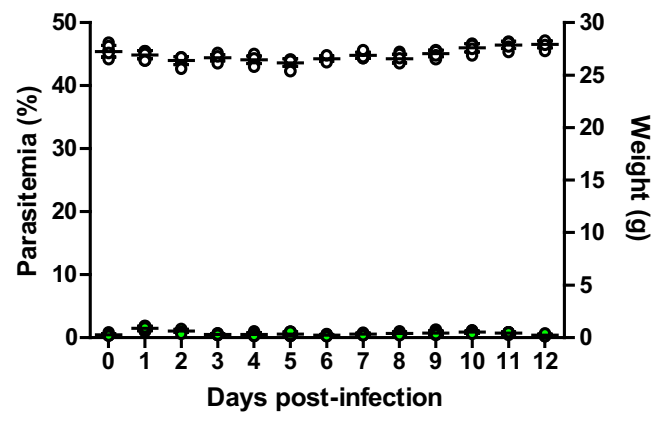

b

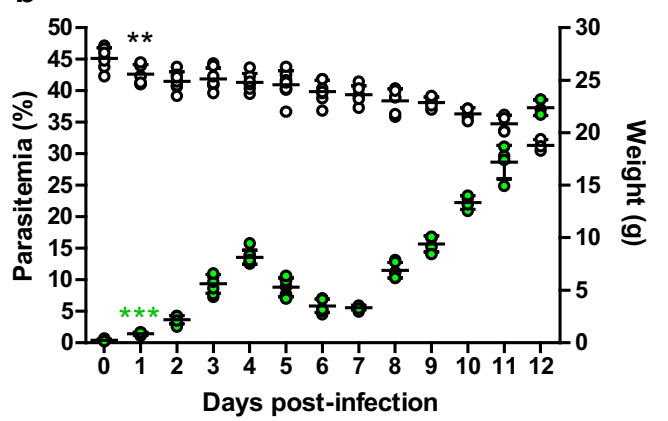

d

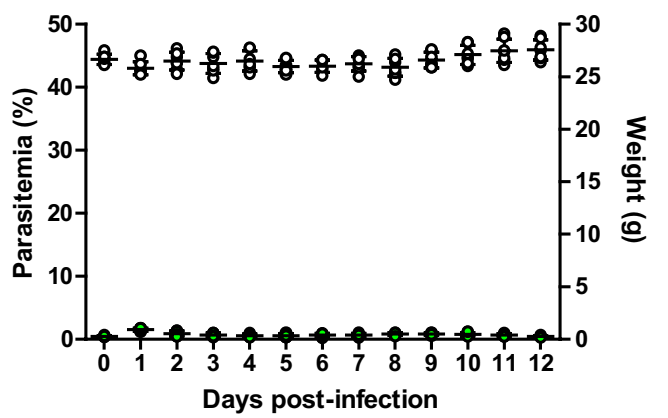

e

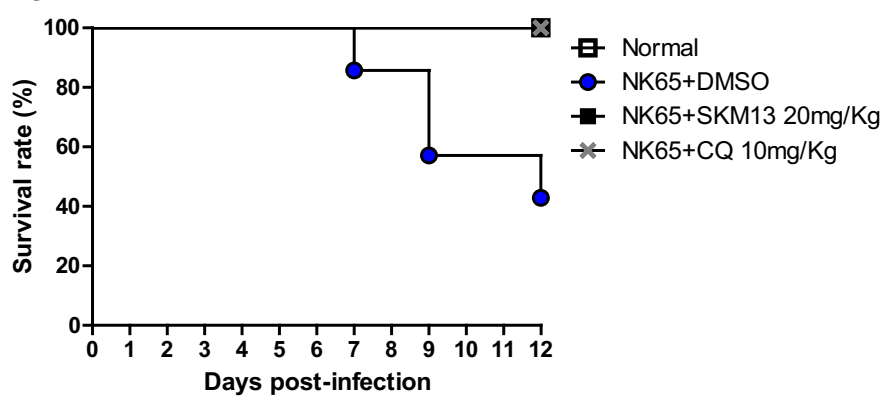

Fig. 2 Curative effect of SKM13 against chloroquine-susceptible strain P. berghei in a 4-day suppression test. Plasmodium berghei (NK65) parasites $\left(10^{7} /\right.$ mouse) were injected via the intraperitoneal (i.p.) route in mice $(n=7)$. After 1 day, drugs were administered via mouse vein for four consecutive days. After drug treatment, parasitaemia and weight were measured every day (a normal group, b $P$. berghei-infected group, c SKM13-treated group, d CQ-treated group; green circle, parasitaemia; open circle, body weight) ${ }^{* *} P<0.01$, $\left.{ }^{* * *} P<0.001\right)$. The values shown are the mean \pm SD. The survival rate (\% of control) is shown in (e)

\section{Discussion}

The present study demonstrated that hybrid molecules with a CQ structural template, including a quinolone moiety and a modified side chain such as the $\alpha, \beta$ unsaturated amides, could be a useful strategy in the development of anti-malarial drugs.

Because malarial disease is exacerbated by the emergence of drug resistance to efficient treatments, novel anti-malarial medicines are an essential component of malaria control; a continuous search for different drugs with novel chemical structures and mechanism of actions is necessary [31]. As a new strategy in drug design, the hybrid molecule has been characterized by the rational approach of anti-malarial drug design and considered as a method to optimize the therapy with available drugs [32].

One type of these hybrid molecules, quinoline-novel target based hybrid molecules, is widely indicated, because the quinoline entity can inhibit $\beta$-haematin formation and contribute to a remarkable drug concentration within the digestive vacuole of Plasmodium [33]. So far, a quinoline-based isatin derivative, with the quinoline and isatin groups able to inhibit cysteine proteases, has been reported to show in vitro anti-plasmodial activity [34]. However, the in vivo anti-plasmodial activity of the quinoline-based isatin derivative has not yet been elucidated.

In this study, previous finding that the Michael-acceptor of the $\alpha, \beta$-unsaturated amide could form a covalent 

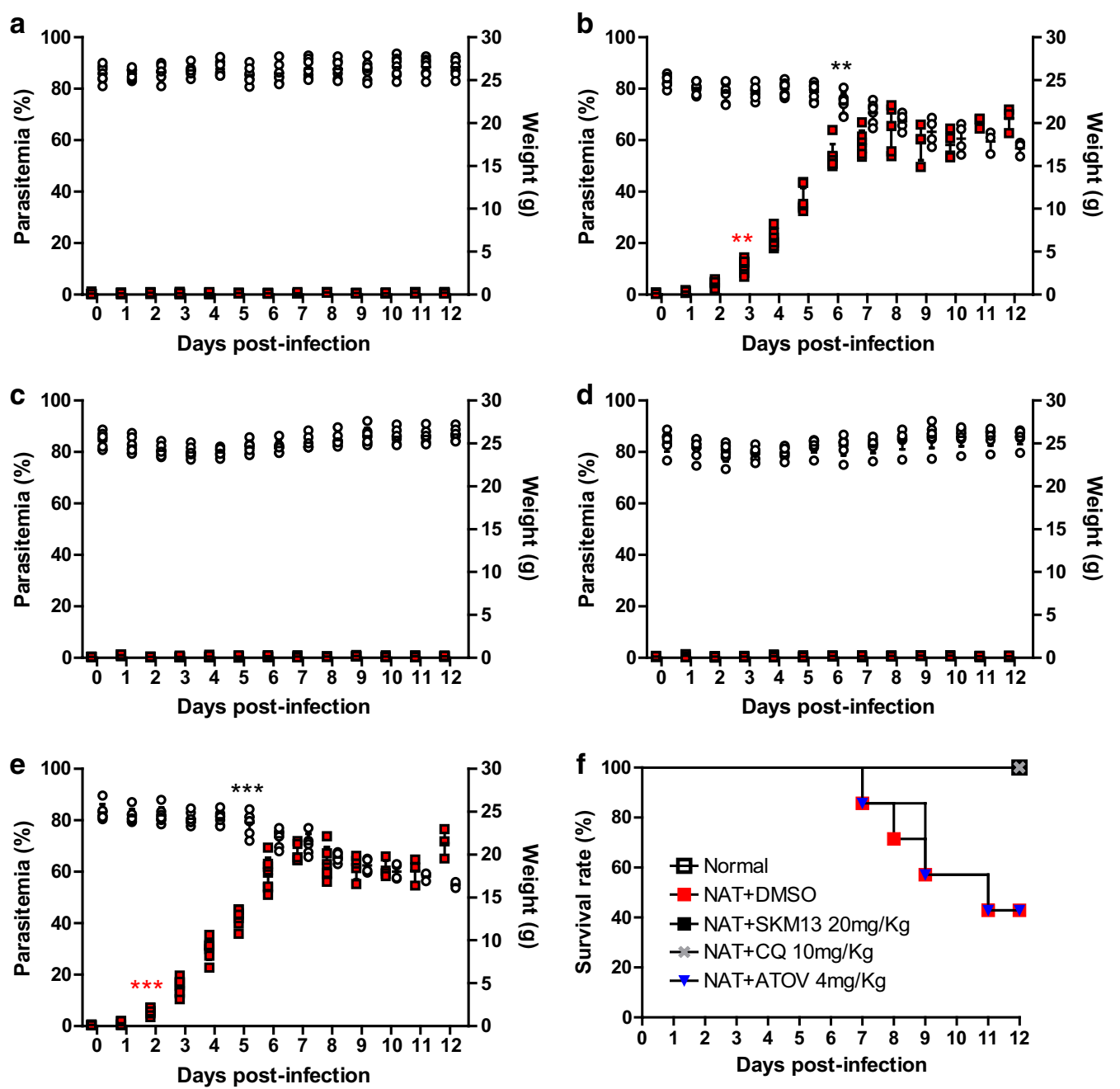

Fig. 3 Effect of SKM13 against chloroquine-resistant strain P. berghei in a 4-day suppression test. Plasmodium berghei (NAT) parasites ( $\left.10^{7} / \mathrm{mouse}\right)$ were injected via the i.p. route in mice $(n=7)$ and after 1 day, drugs were administered in the mouse vein for four consecutive days. After drug treatment, parasitaemia and weight were measured every day (a normal group, b $P$. berghei-infected group, c SKM13-treated group, d CQ-treated group, e atovaquone-treated group; red square, parasitaemia; white circle, body weight) ( $\left.{ }^{* *} P<0.01,{ }^{* * *} P<0.001\right)$. The values shown are the mean \pm SD. The survival rate (\% of control) is shown in (f)

bond between the thiolate in a cysteine protease and the $\beta$ carbon of the $\alpha, \beta$-unsaturated amide [22] was combined with quinoline as a multi-therapeutic strategy, leading to novel CQ derivatives (SKM13 and SKM14). CQ has a small methyl group on the side chain but SKM13 has a phenylmethyl group derived from phenylalanine. In our result, SKM13 with a bigger substituent than methyl group in CQ showed better inhibitory activity, implicating that it may be preferable to develop derivatives with various larger substituents. Despite the structural similarities of the phenylalanine derivatives SKM13 and SKM14, the selective index of SKM13 was two-fold higher than SKM14. The $\alpha, \beta$-unsaturated amide in SKM13 is shorter than that in SKM14. From the results, two effects of the structure of the derivative on the activity of the drug were estimated. First, although the alanine derivative is a similar size to chloroquine, the much larger phenyl group appeared to offer better binding to the target protein. Therefore, the possibility for the use of an amino acid with a larger residue than alanine could be the better strategy for anti-malarial design. Second, as shown by the anti-malarial activity of the derivatives with slightly different alkyl groups located at the nitrogen atom of the amide, the alkyl group could be tuned by size, bulkiness, and polarity.

SKM13 was identified as a potential anti-malarial drug by the complete clearance of parasitaemia, $100 \%$ survival rate, and unchanged haematological features in the 4-day suppression test. Specifically, SKM13 was a more efficient anti-malarial candidate than SKM14, implying that 


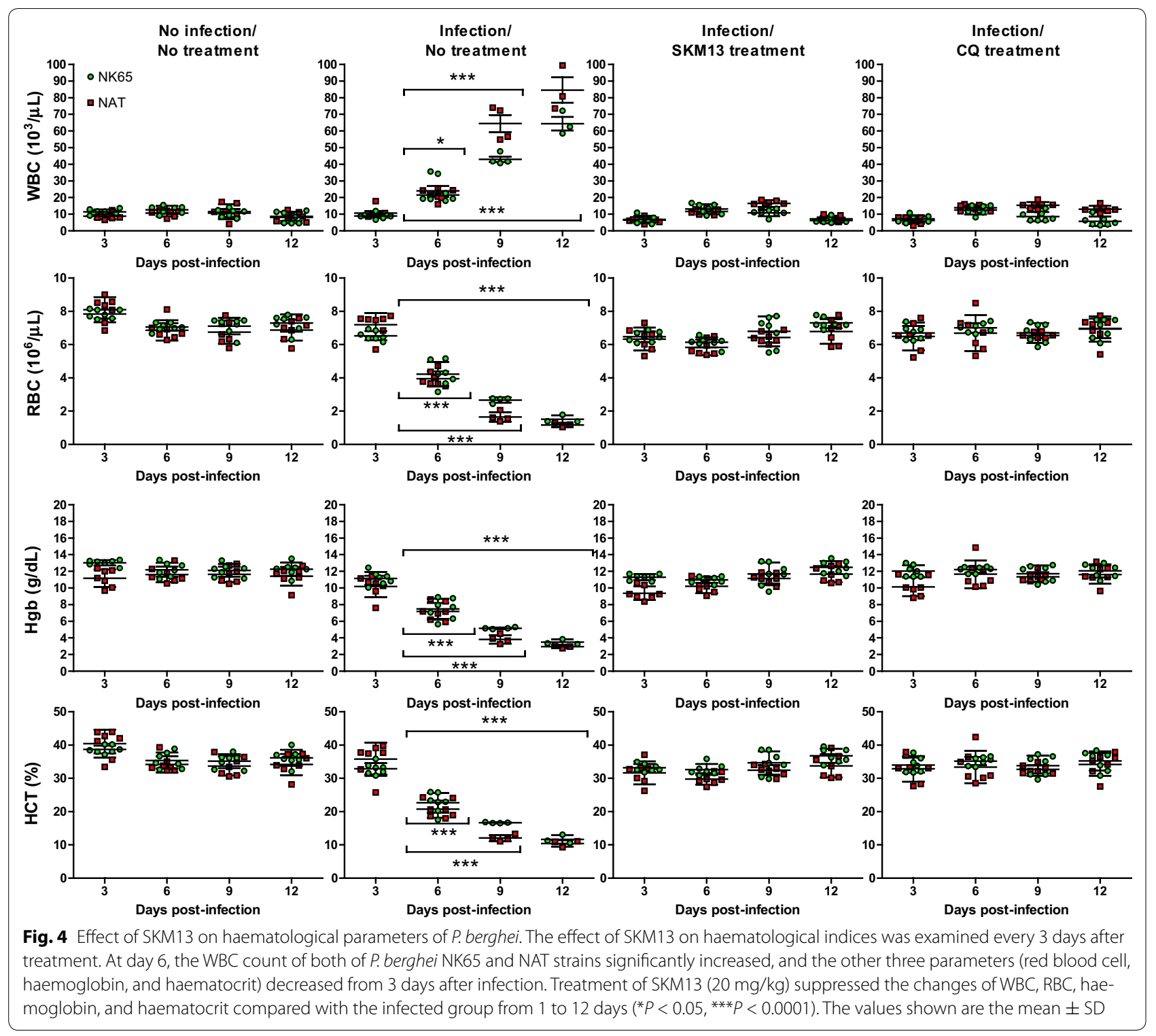

$\alpha, \beta$-unsaturated amides may be an important strategy for anti-malarial drug design.

Haematological parameters, such as red blood cells, leukocytes, and thrombocytes, are considered a biomarker of malarial infection and frequently monitored as an indicator of drug efficacy against Plasmodium infection. Typically, WBC counts were significantly higher in patients with high parasitaemia than in those with low and moderate parasitaemia [29]. Leukocyte counts, especially of neutrophils, have been reported significantly higher in patients with high parasitaemia compared to that in those with low parasitaemia, which supports the high WBC counts with high parasitaemia in the current rodent model.
Changes in RBC are the most typical feature of malarial infections and the most common complication during malarial infection is anaemia [35]. In the present study, more than $25 \%$ loss of total RBC, haemoglobin, and haematocrit demonstrated severe anaemia, and was observed at $6 \mathrm{dpi}$ in both CQ-susceptible and P. berghei(NK65)- and atovaquone-resistant P. berghei (NAT)infected mice. The superior efficacy of SKM13 was supported by the absences of changes in the total RBC, haemoglobin, and haematocrit, which indicated 100\% parasite clearance. Therefore, it is considered that SKM13 is one of the compounds that can suppress the potential malarial burden. 
Based on an in vitro study, SKM13 may not be as effective as CQ in CQ-susceptible $P$. falciparum, but may be a potentially attractive drug in CQ-resistant $P$. falciparum, which indicates that the mechanism of action may be different between CQ and SKM13.

The limitations of this study included the absence of in vivo results derived from CQ-resistant strain-infected mice. The study of CQ-resistant strain-infected mice will help the interpretation of the efficacy of SKM13. Future elucidation of the mode of action of SKM13 would be necessary for it to be considered as a novel strategy for anti-malarial drug design.

\section{Conclusions}

SKM13 with substituents such as phenylmethyl group and the $\alpha, \beta$-unsaturated amides was more effective than CQ against the CQ-resistant strain $P$. falciparum. SKM13 treatment $(20 \mathrm{mg} / \mathrm{kg})$ inhibited the $P$. berghei growth completely in the blood of mice and increased the mice survival rate from 40 to $100 \%$. Haematological parameters supported the in vivo efficacy of SKM13 for 4-day suppression test. In conclusion, CQ derivatives with substituents such as the $\alpha, \beta-$ unsaturated amides and phenylmethyl group can be a useful strategy for improving the anti-malarial activity and SKM13 is an excellent candidate as anti-malarial drug.

\section{Additional file}

Additional file 1: Figure S1. FACS analysis for $\mathrm{IC}_{50}$ of $\mathrm{CQ}$ in P. falciparum (3D7). Figure S2. FACS analysis for $I C_{50}$ of SKM13 in P. falciparum (3D7). Figure S3. FACS analysis for $\mathrm{IC}_{50}$ of SKM14 in P. falciparum (3D7). Figure S4. $F A C S$ analysis for $I C_{50}$ of $C Q$ in $P$. falciparum (FCR3). Figure S5. FACS analysis for $\mathrm{IC}_{50}$ of SKM13 in P. falciparum (FCR3). Figure S6. FACS analysis for $\mathrm{IC}_{50}$ of SKM14 in P. falciparum (FCR3).

\section{Authors' contributions}

SJ and DX carried out the assays and analyzed the results; SJ, HS and HP wrote the manuscript; HS and HP conceived and designed the experiments. All authors read and approved the final manuscript.

\section{Acknowledgements}

This research was supported by Priority Research Centers Program through the National Research Foundation of Korea (NRF) funded by the Ministry of Education, (NRF-2015R1A6A1A03032236), and a National Research Foundation of Korea (NRF) Grant funded by the Korean government (NRF-2015R1D1A4A01020393).

\section{Competing interests}

The authors declare that they have no competing interests.

\section{Availability of data and materials}

All synthesized materials (SKM13 and SKM14) are available in 1 month.

\section{Ethics approval and consent to participate}

Animal experiments were performed at using the experimental protocol which was approved by the Animal Care and Use Committee at Wonkwang University (WKU16-40).
Received: 8 December 2016 Accepted: 6 February 2017

Published online: 17 February 2017

\section{References}

1. Roll Back Malaria. World malaria report 2005. Geneva: World Health Organization and UNICEF; 2005. http://www.who.int/malaria/ publications/atoz/9241593199/en/.

2. CDC. Malaria; 2015. https://www.cdc.gov/malaria/.

3. Schellenberg $D$, Abdulla $S$, Roper C. Current issues for anti-malarial drugs to control P. falciparum malaria. Curr Mol Med. 2006;6:253-60.

4. Slater AF. Chloroquine: mechanism of drug action and resistance in Plasmodium falciparum. Pharmacol Ther. 1993;57:203-35.

5. Achan J, Talisuna AO, Erhart A, Yeka A, Tibenderana JK, Baliraine FN, et al. Quinine, an old anti-malarial drug in a modern world: role in the treatment of malaria. Malar J. 2011;10:144.

6. CDC. Drug resistance in the malaria-endemic world; 2015. https://www. cdc.gov/malaria/malaria_worldwide/reduction/drug_resistance.html.

7. Diagana TT. Supporting malaria elimination with 21 st century anti-malarial agent drug discovery. Drug Discov Today. 2015;20:1265-70.

8. Chou AC, Chevli R, Fitch CD. Ferriprotoporphyrin IX fulfills the criteria for identification as the chloroquine receptor of malaria parasites. Biochemistry. 1980;19:1543-9.

9. Saliba KJ, Folb PI, Smith PJ. Role for the Plasmodium falciparum digestive vacuole in chloroquine resistance. Biochem Pharmacol. 1998:56:313-20.

10. Savarino A, Boelaert JR, Cassone A, Majori G, Cauda R. Effects of chloroquine on viral infections: an old drug against today's diseases? Lancet Infect Dis. 2003:3:722-7.

11. Yan Y, Zou Z, Sun Y, Li X, Xu KF, Wei Y, Jin N, Jiang C. Anti-malaria drug chloroquine is highly effective in treating avian influenza $A$ H5N1 virus infection in an animal model. Cell Res. 2013;23:300-2.

12. Sotelo J, Briceno E, Lopez-Gonzalez MA. Adding chloroquine to conventional treatment for glioblastoma multiforme: a randomized, doubleblind, placebo-controlled trial. Ann Intern Med. 2006:144:337-43.

13. Chen PM, Gombart ZJ, Chen JW. Chloroquine treatment of ARPE-19 cells leads to lysosome dilation and intracellular lipid accumulation: possible implications of lysosomal dysfunction in macular degeneration. Cell Biosci. 2011;1:10.

14. Hawkes M, Conroy AL, Kain KC. Spread of artemisinin resistance in malaria. N Engl J Med. 2014;371:1944-5.

15. Ajayi NA, Ukwaja KN. Possible artemisinin-based combination therapyresistant malaria in Nigeria: a report of three cases. Rev Soc Bras Med Trop. 2013:46:525-7.

16. Malhotra R, Mutreja D. A case of complicated falciparum malaria with treatment failure to artemisinin combination therapy. J Assoc Physicians India. 2014;62:861-3.

17. Andrews S, Burgess SJ, Skaalrud D, Kelly JX, Peyton DH. Reversal agent and linker variants of reversed chloroquines: activities against Plasmodium falciparum. J Med Chem. 2010;53:916-9.

18. Ekoue-Kovi K, Yearick K, Iwaniuk DP, Natarajan JK, Alumasa J, de Dios AC, Roepe PD, Wolf C. Synthesis and anti-malarial activity of new 4-amino7-chloroquinolyl amides, sulfonamides, ureas and thioureas. Bioorg Med Chem. 2009;17:270-83.

19. Ryckebusch A, Debreu-Fontaine MA, Mouray E, Grellier P, Sergheraert C, Melnyk P. Synthesis and anti-malarial evaluation of new N1-(7-chloro4-quinolyl)-1,4-bis(3-aminopropyl)piperazine derivatives. Bioorg Med Chem Lett. 2005; 15:297-302.

20. Russo I, Oksman A, Vaupel B, Goldberg DE. A calpain unique to alveolates is essential in Plasmodium falciparum and its knockdown reveals an involvement in pre-S-phase development. Proc Natl Acad Sci USA. 2009:106:1554-9.

21. Choi HJ, Cui M, Li DY, Song HO, Kim HS, Park H. Anti-malarial activity of new N-acetyl-L-leucyl-L-leucyl-L-norleucinal (ALLN) derivatives against Plasmodium falciparum. Bioorg Med Chem Lett. 2013;23:1293-6.

22. Mallik SK, Li DY, Cui M, Song HO, Park H, Kim HS. Synthesis and evaluation of peptidyl alpha, beta-unsaturated carbonyl derivatives as anti-malarial calpain inhibitors. Arch Pharm Res. 2012:35:469-79. 
23. Cheng J, Zeidan R, Mishra S, Liu A, Pun SH, Kulkarni RP, Jensen GS, Bellocq NC, Davis ME. Structure-function correlation of chloroquine and analogues as transgene expression enhancers in nonviral gene delivery. J Med Chem. 2006;49:6522-31.

24. Kim J-G, Jang DO. A convenient one-pot method for the synthesis of $\mathrm{N}$-methoxy-N-methyl amides from carboxylic acids. Bull Korean Chem Soc. 2010;31:171-3.

25. Paris M, Pothien C, Goulleux L, Heitz A, Martineza J, Fehrentza J-A. Synthesis of peptide aldehydes on solid support. React Funct Polym. 1999;41:255-61.

26. Ando KA. Mechanistic study of the Horner-Wadsworth-Emmons reaction: computational investigation on the reaction pass and the stereochemistry in the reaction of lithium enolate derived from trimethyl phosphonoacetate with acetaldehyde. J Org Chem. 1999;64:6815-21.

27. Yeo SJ, Huong DT, Han JH, Kim JY, Lee WJ, Shin HJ, et al. Performance of coumarin-derived dendrimer-based fluorescence-linked immunosorbent assay (FLISA) to detect malaria antigen. Malar J. 2014;13:266.

28. Ekengard E, Glans L, Cassells I, Fogeron T, Govender P, Stringer T, et al. Anti-malarial activity of ruthenium(II) and osmium(II) arene complexes with mono- and bidentate chloroquine analogue ligands. Dalton Trans. 2015;44:19314-29.
29. Kotepui M, Piwkham D, PhunPhuech B, Phiwklam N, Chupeerach C, Duangmano $S$. Effects of malaria parasite density on blood cell parameters. PLOS ONE. 2015;10:e0121057.

30. Taconic. Phenotypic data of ICR; 2003. http://www.taconic.com/ mouse-model/icr.

31. Basco LK, Dechy-Cabaret O, Ndounga M, Meche FS, Robert A, Meunier B. In vitro activities of DU-1102, a new trioxaquine derivative, against Plasmodium falciparum isolates. Antimicrob Agents Chemother. 2001;45:1886-8.

32. Muregi FW, Ishih A. Next-generation anti-malarial drugs: hybrid molecules as a new strategy in drug design. Drug Dev Res. 2010;71:20-32.

33. Pagola S, Stephens PW, Bohle DS, Kosar AD, Madsen SK. The structure of malaria pigment beta-haematin. Nature. 2000;404:307-10.

34. Chiyanzu I, Clarkson C, Smith PJ, Lehman J, Gut J, Rosenthal PJ, et al. Design, synthesis and anti-plasmodial evaluation in vitro of new 4-aminoquinoline isatin derivatives. Bioorg Med Chem. 2005;13:3249-61.

35. Erhart LM, Yingyuen K, Chuanak N, Buathong N, Laoboonchai A, Miller RS, et al. Hematologic and clinical indices of malaria in a semi-immune population of western Thailand. Am J Trop Med Hyg. 2004;70:8-14.

\section{Submit your next manuscript to BioMed Central and we will help you at every step:}

- We accept pre-submission inquiries

- Our selector tool helps you to find the most relevant journal

- We provide round the clock customer support

- Convenient online submission

- Thorough peer review

- Inclusion in PubMed and all major indexing services

- Maximum visibility for your research

Submit your manuscript at www.biomedcentral.com/submit 